\title{
Influencia del cobre, estaño, fósforo y arsénico en el agrietamiento de la superficie del acero inoxidable 18-8 durante la compresión a altas temperaturas
}

\author{
J. Botella $^{(*)}$, M.T. Fernández ${ }^{(*)}$ e I. Fernández de Castillo ${ }^{(*)}$
}

\begin{abstract}
Resumen Esta investigación aborda el estudio del efecto de concentraciones variables de cobre, estaño, fósforo y arsénico en el agrietamiento de la superficie de un acero 18-8, sometido a ensayos de compresión, a 1.123 y $1.273 \mathrm{~K}$, en atmósfera oxidante (aire). Se desarrolla un procedimiento de cuantificación del grado de agrietamiento y se relaciona cada índice de agrietamiento así obtenido, a las distintas temperaturas, con la composición química de los materiales. Los índices de agrietamiento correspondientes a los materiales comprimidos a $1.273 \mathrm{~K}$ se comparan con los valores de pérdida de reducción de área obtenidos mediante ensayos de tracción a la misma temperatura.
\end{abstract}

Palabras clave: Acero inoxidable austenítico. Cobre. Estaño. Fósforo. Arsénico. Agrietamiento superficial.

\section{Effect of copper, tin, phosphorous and arsenic on the surface cracking of a 18-8 stainless steel during hot compression tests}

\begin{abstract}
The effect of certain different concentrations of $\mathrm{Cu}, \mathrm{Sn}, \mathrm{P}$ and As on the surface cracking of 18-8 austenitic stainless steel hot compressed specimens has been studied, at 1,123 and $1,273 \mathrm{~K}$, in an oxidizing atmosphere (air). A procedure for determining surface cracking has been established, and the cracking factor obtained in this way is correlated with the chemical composition of the materials at both temperatures. The cracking factors obtained at $1,273 \mathrm{~K}$ have been compared with the reduction of area drops obtained by hot tension tests at the same temperature.
\end{abstract}

Keywords: Austenitic stainless steel. Copper. Tin. Phosphorous. Arsenic. Surface cracking.

\section{INTRODUCCIÓN}

La presencia de elementos tales como $\mathrm{Cu}, \mathrm{Sn}, \mathrm{P}$ y As en la composición química de los aceros inoxidables austeníticos puede provocar fallos de la ductilidad en caliente (1-4), siendo el fenómeno de la formación de astillados y grietas en bordes la manifestación más frecuente y grave desde el punto de vista del deterioro de la calidad de la superficie. Ya que de dicha calidad depende no sólo el aspecto estético, fundamental en el inoxidable, sino también su comportamiento en uso (embutibilidad, resisten-

(*) I+D ACERINOX, S.A., Aptdo. 83, 11379-Los Barrios (Cádiz, España). cia a la corrosión, etc), se plantea la necesidad de establecer criterios que relacionen las concentraciones de los citados elementos con el grado del eventual deterioro de la superficie que provocan. El presente trabajo constituye una contribución en dicho sentido.

\section{MÉTODO EXPERIMENTAL}

\subsection{Materiales del estudio}

Los materiales empleados en este estudio han sido lingotes de $40 \mathrm{~kg}$ fabricados en un horno de inducción de laboratorio. En la tabla I se relacionan las composiciones empleadas: El típico acero 
TABLA. I.- Composición química de los lingotes

TABLE. I.- Chemical composition of the ingots

\begin{tabular}{|c|c|c|c|c|c|c|c|c|c|c|c|c|c|}
\hline & & \multicolumn{10}{|c|}{ \% en masa } & \multicolumn{1}{|c|}{ ppm } \\
\cline { 3 - 13 } Mat. & $\mathrm{C}$ & $\mathrm{Si}$ & $\mathrm{Mn}$ & $\mathrm{Cr}$ & $\mathrm{Ni}$ & $\mathrm{Mo}$ & $\mathrm{S}$ & $\mathrm{Cu}$ & $\mathrm{Sn}$ & $\mathrm{P}$ & $\mathrm{As}$ & $\mathrm{N}$ & $\mathrm{O}$ \\
\hline $18 / 8$ & 0,059 & 0,38 & 1,44 & 18,30 & 8,17 & 0,19 & 0,004 & 0,31 & 0,016 & 0,020 & 0,011 & 409 & 63 \\
1 & 0,060 & 0,37 & 1,46 & 18,25 & 8,23 & 0,19 & 0,005 & $\mathbf{0 , 9 6}$ & 0,022 & 0,030 & 0,013 & 409 & 97 \\
2 & 0,052 & 0,33 & 1,32 & 18,35 & 8,25 & 0,20 & 0,005 & $\mathbf{2 , 0 1}$ & 0,015 & 0,022 & 0,014 & 408 & 80 \\
3 & 0,053 & 0,35 & 1,43 & 18,29 & 8,26 & 0,20 & 0,004 & $\mathbf{3 , 0 4}$ & 0,016 & 0,029 & 0,015 & 417 & 76 \\
4 & 0,058 & 0,35 & 1,45 & 18,23 & 8,19 & 0,18 & 0,006 & 0,37 & $\mathbf{0 , 0 5 1}$ & 0,032 & 0,015 & 404 & 98 \\
5 & 0,058 & 0,34 & 1,43 & 18,37 & 8,25 & 0,20 & 0,005 & 0,41 & $\mathbf{0 , 0 8 0}$ & 0,033 & 0,015 & 387 & 85 \\
6 & 0,056 & 0,37 & 1,43 & 18,20 & 8,21 & 0,20 & 0,005 & 0,35 & $\mathbf{0 , 1 6 0}$ & 0,031 & 0,014 & 398 & 97 \\
7 & 0,055 & 0,35 & 1,42 & 18,24 & 8,18 & 0,20 & 0,005 & 0,30 & 0,016 & $\mathbf{0 , 1 3 8}$ & 0,036 & 417 & 90 \\
8 & 0,055 & 0,38 & 1,45 & 18,35 & 8,26 & 0,18 & 0,004 & 0,25 & 0,017 & 0,040 & $\mathbf{0 , 1 0 4}$ & 382 & 81 \\
9 & 0,058 & 0,37 & 1,44 & 18,45 & 8,29 & 0,20 & 0,005 & 0,29 & 0,014 & 0,038 & $\mathbf{0 , 2 0 5}$ & 402 & 79 \\
\hline
\end{tabular}

inoxidable austenítico 18-8 (AISI 304) se ha designado como colada base, a la cual se han adicionado concentraciones variables de $\mathrm{Cu}, \mathrm{Sn}, \mathrm{P}$ y As, obteniéndose así el resto de los lingotes.

Se ha logrado mantener prácticamente invariable la composición química de los lingotes a excepción de la deseada variación de los elementos en estudio.

\subsection{Preparación de las probetas para los ensayos de compresión. Ciclo termomecánico seguido}

De la zona central del lingote se han cortado probetas de $14 \times 14 \times 35 \mathrm{~mm}$ con el eje longitudinal perpendicular al eje longitudinal del lingote. Estas muestras fueron sometidas al tratamiento térmico seguido por los desbastes industriales previo a la laminación en caliente, consistente en calentamiento lento, mediante una serie de rampas, hasta $1.523 \mathrm{~K}$, seguido de mantenimiento durante $45 \mathrm{~min}$ a esta temperatura. Las probetas fueron enfriadas súbitamente en agua desde dicha temperatura, y posteriormente mecanizadas a probetas cilíndricas de $12 \mathrm{~mm}$ de longitud y $10 \mathrm{~mm}$ de diámetro (Fig.1), empleadas en los ensayos de compresión en caliente.

Los ensayos de compresión se han llevado a cabo en el simulador de ensayos termomecánicos Gleeble 1500 en aire, a 1.273 y $1.123 \mathrm{~K}$. El ciclo termomecánico empleado consta de las siguientes etapas: Calentamiento a una velocidad de $299 \mathrm{~K} / \mathrm{s}$ hasta alcanzar $1.473 \mathrm{~K}$, mantenimiento a $1.473 \mathrm{~K}$ durante $10 \mathrm{~min}$, enfriamiento rápido con velocidad de $293 \mathrm{~K} / \mathrm{s}$ a las temperaturas de ensayo 1.273 ó $1.123 \mathrm{~K}$; una vez alcanzada la temperatura deseada, se aplican seis pasadas de compresión, sin tiempo de espera, con reducciones comprendidas entre 18 y 22 $\%$, pasando de un espesor inicial de $12 \mathrm{~mm}$ al espe-

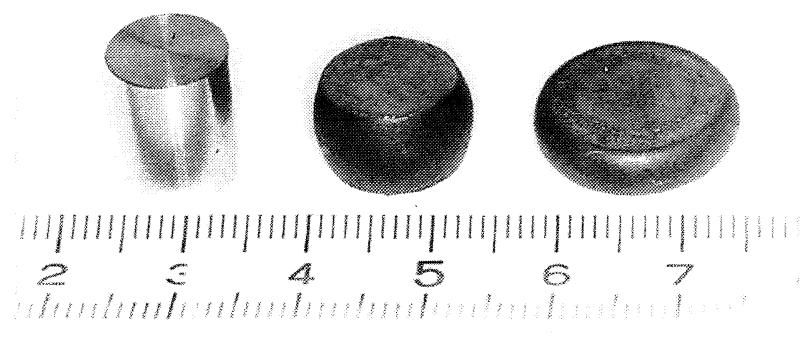

FIG. 1.- Sucesión de pasos de deformación.

FIG. 1.-Different reduction steps.

sor final de $\simeq 3,3 \mathrm{~mm}$, lo que equivale a obtener una reducción total de $72,5 \%$ aproximadamente.

\subsection{Estimación del agrietamiento de la superficie de los materiales comprimidos}

La figura 2 presenta, como ejemplo, la superficie lateral agrietada de uno de los materiales. La
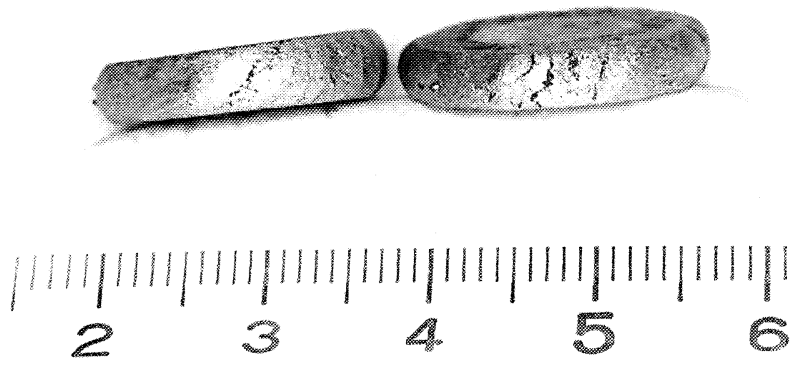

FIG. 2.- Superficie agrietada a: (izq.) $1.273 \mathrm{~K} \mathrm{y}$ (dcha.) $1.123 \mathrm{~K}$.

FIG. 2.-Cracking surface at (left) $1,273 \mathrm{~K}$ and (right) $1,123 \mathrm{~K}$. 
observación de la superficie deformada de las probetas se ha realizado mediante lupa de 10 aumentos con escala graduada en milímetros, determinándose el número, longitud y ancho de las grietas formadas en el centro y borde de dicha superficie.

El agrietamiento de los materiales se ha establecido mediante el parámetro denominado índice de agrietamiento $(I A)=L \times a$, expresado en $\mathrm{mm}^{2}$, siendo $L$ la longitud total de las grietas presentes en la superficie, en mm, y $a$ el ancho medio de las grietas, en $\mathrm{mm}$. Atendiendo a los valores de índice de agrietamiento asignados a los distintos materiales ensayados, se han definido una serie de intervalos que dan cuenta del deterioro de la calidad superficial de estos materiales:

1) Agrietamiento leve de la superficie para valores de $I A \leq 2 \mathrm{~mm}^{2}$.

2) Agrietamiento moderado de la superficie cuando $2<I A \leq 4 \mathrm{~mm}^{2}$.

3) Agrietamiento elevado de la superficie si $I A \geq 4$ $\mathrm{mm}^{2}$.

\section{RESULTADOS Y DISCUSIÓN}

Las figuras 3 y 4 presentan las variaciones de los índices de agrietamiento frente a las concentraciones de $\mathrm{Cu}, \mathrm{Sn}, \mathrm{P}$ y As a las dos temperaturas de ensayo.

Respecto de los ensayos realizados a $1.273 \mathrm{~K}$ (Fig. 3), el inoxidable 18-8, así como los lingotes aleados con $0,96 \% \mathrm{Cu}$ y $0,05 \% \mathrm{Sn}$, con índices de agrietamiento $<2 \mathrm{~mm}^{2}$, muestran un leve deterioro de la superficie.

Los materiales dopados con concentraciones de hasta $2,01 \% \mathrm{Cu}, 0,08 \% \mathrm{Sn}, 0,14 \%$ P y $0,10 \%$ As

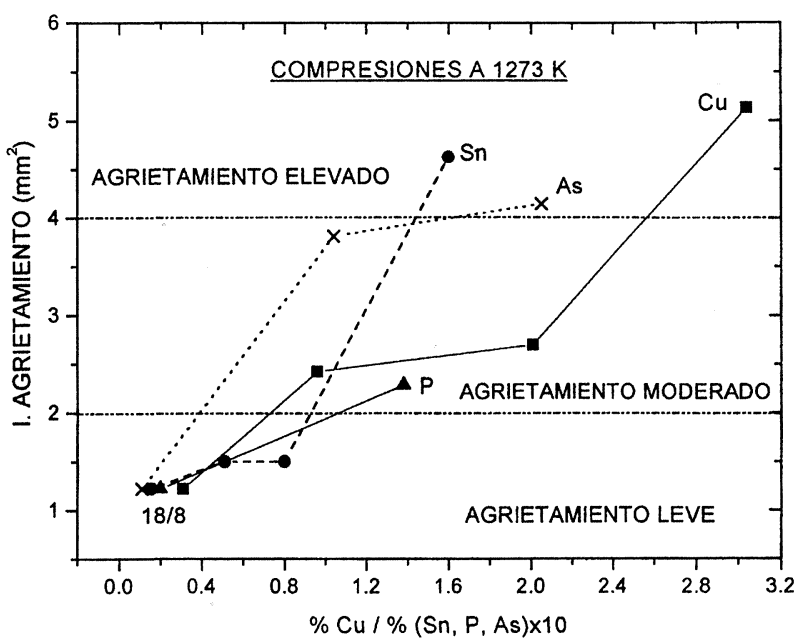

FIG. 3.- Índice de agrietamiento $v s \%(\mathrm{Cu}, \mathrm{Sn}, \mathrm{P}$, As) a $1.273 \mathrm{~K}$.

FIG. 3.- Cracking factor vs $(\mathrm{Cu}, \mathrm{Sn}, \mathrm{P}, \mathrm{As}) \%$ at $1,273 \mathrm{~K}$.

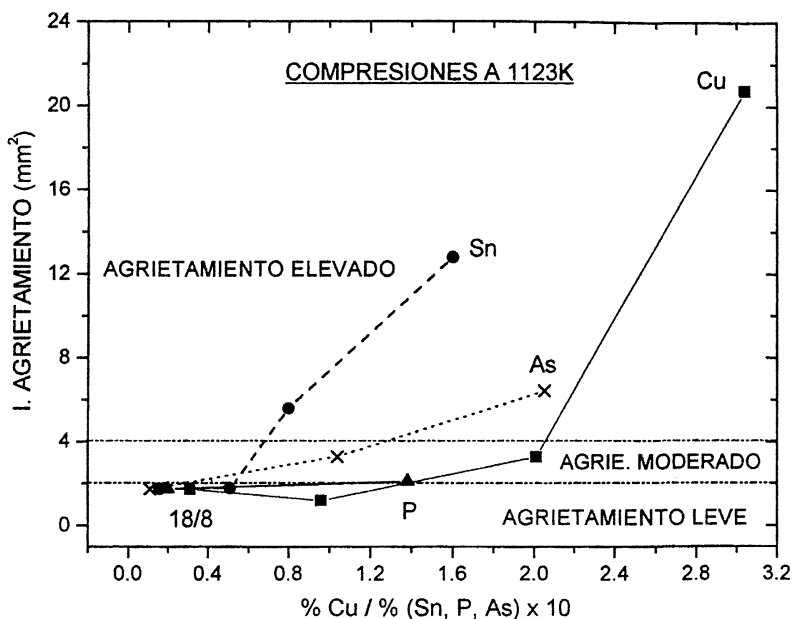

FIG. 4. - Índice de agrietamiento $v s \%(\mathrm{Cu}, \mathrm{Sn}, \mathrm{P}$, As) a $1.123 \mathrm{~K}$.

FIG. 4.- Cracking factor vs $(\mathrm{Cu}, \mathrm{Sn}, \mathrm{P}, \mathrm{As}) \%$ at $1,123 \mathrm{~K}$.

presentan índices de agrietamiento entre 2 y $4 \mathrm{~mm}^{2}$, lo que implica un agrietamiento moderado de la superficie. Los lingotes más dopados: $3,04 \% \mathrm{Cu}$, $0,16 \%$ Sn y $0,21 \%$ As presentan superficies severamente agrietadas.

A $1.123 \mathrm{~K}$ (Fig. 4) continúan mostrando un bajo índice de agrietamiento, tanto el clásico inoxidable 18-8 como las coladas menos dopadas con $\mathrm{Cu}, \mathrm{Sn}, \mathrm{P}$ y As. Las coladas más dopadas presentan ahora una superficie más deteriorada que a $1.273 \mathrm{~K}$ : Contenidos del 3,04 \% Cu cuadruplican el grado de agrietamiento, en tanto que $0,16 \%$ Sn lo triplican y $0,21 \%$ As deterioran la superficie 1,6 veces más que a 1.273 $\mathrm{K}$. El inoxidable dopado con $0,08 \%$ Sn presenta una superficie más agrietada a $1.123 \mathrm{~K}$ que a $1.273 \mathrm{~K}$.

En la figura 5 se compara el deterioro de la superficie de los materiales comprimidos a las dos

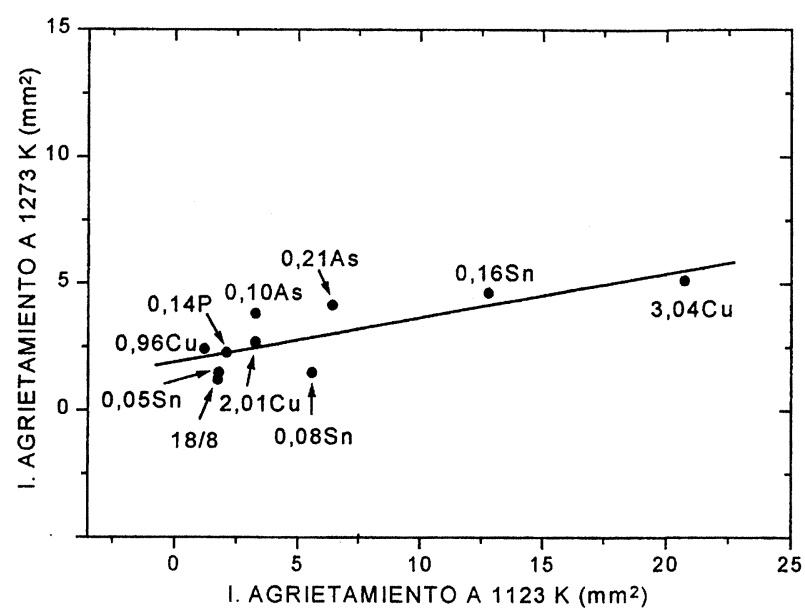

FIG. 5.- Índice de agrietamiento a $1.273 \mathrm{~K} v s$ $1.123 \mathrm{~K}$.

FIG. 5.-Cracking factor at $1,273 \mathrm{~K}$ vs $1,123 \mathrm{~K}$. 
temperaturas de ensayo. Se observa que los inoxidables dopados con concentraciones de hasta 2,01 $\% \mathrm{Cu}, 0,05 \% \mathrm{Sn}, 0,14 \% \mathrm{P}$ y $0,10 \%$ As muestran similares índices de agrietamiento tanto a 1.273 como a $1.123 \mathrm{~K}$. Concentraciones del orden de 0,08 $\%$ Sn y $0,21 \%$ As incrementan ligeramente el deterioro de la superficie del inoxidable ensayado a $1.123 \mathrm{~K}$, mientras que, como se ha descrito anteriormente, las aleaciones con $3,04 \% \mathrm{Cu}$ y $0,16 \%$ $\mathrm{Sn}$ sufren graves efectos superficiales a $1.123 \mathrm{~K}$.

Los análisis metalográficos de las secciones transversales de las probetas comprimidas permiten establecer que a $1.273 \mathrm{~K}$ predominan las grietas pequeñas; las grietas mayores, aunque escasas, no superan los $0,5 \mathrm{~mm}$ de profundidad. La mayoría de las grietas se propagan intergranularmente.

A $1.123 \mathrm{~K}$ aparecen grietas bastante profundas, de 2 a $3 \mathrm{~mm}$, sobre las que no es posible predecir el origen de su propagación. Las grietas más pequeñas tienen carácter intergranular.

En la figura 6 se comparan los índices de agrietamiento correspondientes a los materiales comprimidos a $1.273 \mathrm{~K}$ con los valores de porcentaje de pérdida de reducción de área (\% PRA), obtenidos mediante ensayos de tracción a esta misma temperatura (4).

El aumento de la pérdida de reducción de área y del índice de agrietamiento son, en general, proporcionales al aumento de las concentraciones de los elementos $\mathrm{Cu}, \mathrm{Sn}, \mathrm{P}$ y As, estableciéndose la siguiente correlación lineal entre ambos factores [1], con un grado de correlación $R^{2}=0,90$ :

$$
P R A=8,862+9,165 \times I A
$$

Atendiendo a esta regresión lineal y considerando un valor límite de pérdida de reducción de área aceptable: $25 \%$ PRA, se obtiene, cortando la línea

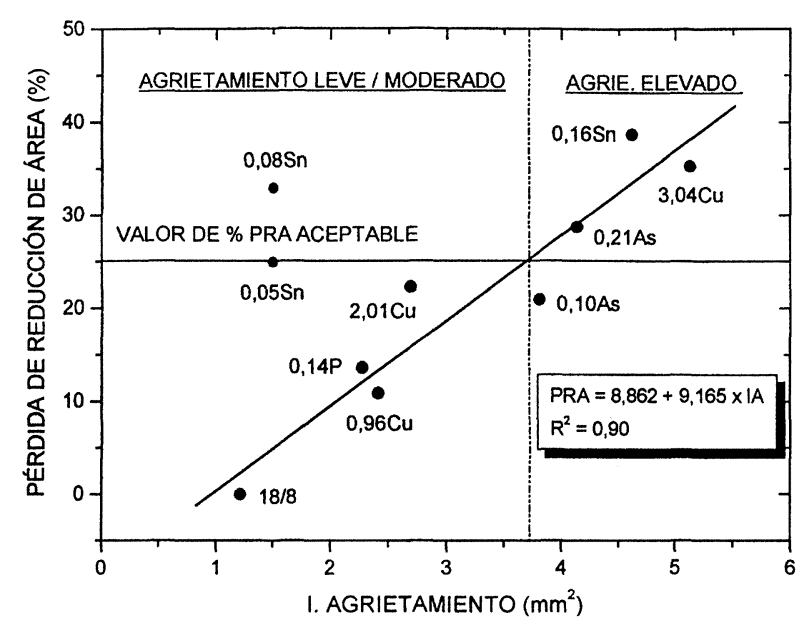

FIG. 6.- Pérdidas de reducción de área $v s$ Índice de agrietamiento a $1.273 \mathrm{~K}$.

FIG. 6.- Reduction of area drops vs Cracking factors at 1,273 K. de regresión, un valor de agrietamiento $\simeq 3,7 \mathrm{~mm}^{2}$, a partir del cual se deteriora gravemente la calidad de la superficie de los materiales.

Se apartan de la tendencia anteriormente definida las coladas dopadas con relativamente altas concentraciones de estaño $(0,05 \%$ y $0,08 \%)$, que presentan pérdidas de reducción de área considerables (25 y $35 \%$, respectivamente) y muestran, por el contrario, una superficie poco agrietada. Se deduce que el estaño, para estas concentraciones, afecta de forma más negativa a la ductilidad interna del material, evidencia puesta de manifiesto en los ensayos de tracción en caliente.

Cabe resaltar que contenidos del $0,14 \%$ P y 0,96 $\% \mathrm{Cu}$ afectan levemente a la ductilidad del inoxidable 18-8, en cuanto a pérdidas de reducción de área y respecto del agrietamiento de la superficie.

\section{CONCLUSIONES}

Los materiales ensayados a compresión uniaxial a 1.273 y $1.123 \mathrm{~K}$ se evalúan desde el punto de vista del agrietamiento de la superficie deformada, estableciéndose el parámetro denominado índice de agrietamiento: $I A=L \times a$, expresado en $\mathrm{mm}^{2}$.

Los lingotes de inoxidable 18-8 menos dopados, $0,96 \% \mathrm{Cu}, 2,01 \% \mathrm{Cu}, 0,05 \% \mathrm{Sn}$ y $0,10 \% \mathrm{As}$, presentan agrietamientos similares a la colada base a ambas temperaturas de ensayo. Muestra este mismo comportamiento la colada con máximo contenido de fósforo $(0,14 \%)$, lo que implica un leve o moderado agrietamiento de la superficie.

Los lingotes más dopados, 3,04\% Cu, 0,16\% Sn y $0,21 \%$ As, muestran superficies más deterioradas que el acero inoxidable base, agravándose el deterioro cuando las compresiones se realizan a $1.123 \mathrm{~K}$. Sigue este mismo comportamiento la colada con contenido medio de estaño $(0,08 \%)$, lo que implica un severo agrietamiento de la superficie.

Existe correlación lineal entre el grado de agrietamiento de la superficie y las pérdidas de reducción de área a $1.273 \mathrm{~K}$, en sentido creciente al incremento de las concentraciones de los elementos en estudio. Se apartan de esta tendencia las coladas menos dopadas con estaño $(0,05 \%$ y $0,08 \% \mathrm{Sn})$ que presentan pronunciadas pérdidas de reducción de área frente a un leve deterioro de la calidad de la superficie.

\section{REFERENCIAS}

(1) Nicholson, A. Iron Steel, 1964: 363-367.

(2) Pruger, T.A., Blake, F. y Valley, J.A. ASTM STP 418, 1967: 24.

(3) Bywater, K.A. y Gladman, T. Metals Technol., 1976: 358-365.

(4) Botella, J. Comisión de las Comunidades Europeas. Infome EUR 13316, 1991.

(5) Peckner, D. y Bernstein, I.M. Handbook of Stainless Steels. McGraw Hill. Cap. 4. EE.UU. 1997. 